\title{
ZEUS: Analyzing Safety of Smart Contracts
}

\author{
Sukrit Kalra \\ IBM Research \\ sukrit.kalra@in.ibm.com
}

\author{
Seep Goel \\ IBM Research \\ sgoel219@in.ibm.com
}

\author{
Mohan Dhawan \\ IBM Research \\ mohan.dhawan@in.ibm.com
}

\author{
Subodh Sharma \\ IIT Delhi \\ svs@iitd.ac.in
}

\begin{abstract}
A smart contract is hard to patch for bugs once it is deployed, irrespective of the money it holds. A recent bug caused losses worth around $\$ 50$ million of cryptocurrency. We present ZEUS - a framework to verify the correctness and validate the fairness of smart contracts. We consider correctness as adherence to safe programming practices, while fairness is adherence to agreed upon higher-level business logic. ZEUS leverages both abstract interpretation and symbolic model checking, along with the power of constrained horn clauses to quickly verify contracts for safety. We have built a prototype of ZEUS for Ethereum and Fabric blockchain platforms, and evaluated it with over 22.4K smart contracts. Our evaluation indicates that about $94.6 \%$ of contracts (containing cryptocurrency worth more than $\$ 0.5$ billion) are vulnerable. ZEUS is sound with zero false negatives and has a low false positive rate, with an order of magnitude improvement in analysis time as compared to prior art.
\end{abstract}

\section{INTRODUCTION}

Blockchain is the design pattern that underpins the Bitcoin cryptocurrency [70]. However, its use of consensus to validate interaction amongst participant nodes is a key enabler for applications that require mutually distrusting peers to conduct business without the need for a trusted intermediary. One such use is to enable a smart contract, which programatically encodes rules to reflect any kind of multi-party interaction. With over $\$ 1.4$ billion invested in blockchain last year [3], and the increasing trend towards autonomous applications, smart contracts are fast becoming the preferred mechanism to implement financial instruments (e.g., currencies, derivatives, wallets, etc.) and applications such as decentralized gambling.

While the faithful execution of a smart contract is enforced by the blockchain's consensus protocol, it remains the prerogative of the participating entities to (i) verify the smart contract's correctness, i.e., the syntactic implementation follows the best practices, and (ii) validate its fairness, i.e., the code adheres to the agreed upon higher-level business logic for interaction. While manual auditing of contracts for correctness is possible to an extent, it still remains laborious and error prone. Automatic formal auditing, on the other hand, requires specialized tools and logic. The problem is exacerbated by the fact that smart contracts, unlike other distributed systems code, are immutable and hard to patch in case of bugs, irrespective of

Network and Distributed Systems Security (NDSS) Symposium 2018 18-21 February 2018, San Diego, CA, USA ISBN 1-891562-49-5

http://dx.doi.org/10.14722/ndss.2018.23082

www.ndss-symposium.org

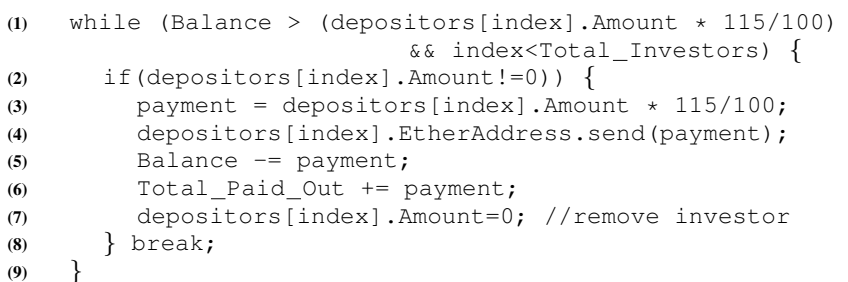

Fig. 1: An unfair contract (adapted from [31]).

the money they hold. For example, investors in TheDAO [43] lost cryptocurrency worth around $\$ 50$ million because of a bug in the code that allowed an attacker to repeatedly siphon off money [44]. In this paper, we tackle the problem of formal verification of smart contracts, since reasoning about their correctness and fairness is critical before their deployment.

The smart contract in Fig. 1 advertises a 15\% profit payout to any investor. However, the contract has both correctness and fairness issues. First, the arithmetic operation in line 6 can potentially overflow, which is a correctness bug. Second, the variable index never increments within the loop, and thus the payout is made to just one investor. Finally, the break statement exits the loop after payment to the first investor, who is the contract owner. Thus, the contract does not payback any other investor. The last two bugs result in fairness issues.

Most prior art in the area of smart contracts deals with security and/or privacy concerns in designing them [62], [64], [67], [73]. There is, however, little work that analyzes smart contracts for vulnerabilities [50], [54], [68]. Oyente [68] uses symbolic execution for bug detection at the bytecode level, but it is neither sound nor complete. Thus, it can result in several false alarms even in trivial contracts, as we observed and communicated to Oyente's developers [26]. Since it is very hard to recreate the intent from the bytecode alone (due to loss of contextual information such as types, reuse of same bytecode for different function calls, etc.) several fairness and correctness issues, including integer overflow/underflow amongst others, are thus completely ignored by Oyente. Further, it conservatively handles loops ${ }^{1}$ [29], [32] (with a bound of one) resulting in under approximation of loop behavior, and thus fails to detect the two fairness bugs in Fig. 1 .

Bhargavan et al. [54] propose a framework to formally verify smart contracts written in a subset of Solidity using $\mathrm{F}^{\star}$, which leaves out important constructs, such as loops. Considering the 22,493 contracts that we analyzed, around 93\% contained loops. Thus, their tool will operate on a fraction of publicly available contracts, which is also corroborated by

\footnotetext{
${ }^{1}$ Oyente is under active development and future releases could add more features and reduce the false alarms.
} 
their results; they could evaluate only 46 out of 396 contracts. While use of $\mathrm{F}^{\star}$ may enable reasoning about most correctness and fairness properties, the authors suggest that such reasoning may require manual proofs. Although it is unclear when such situations may arise. In contrast, we establish that completely automated verification enables analysis of published contracts at a much larger scale. Why3 [50] is an experimental tool for formal verification of Solidity contracts, which is under active development and supports only a small subset of the entire syntax [22]. Further, Solidity to Why3 translation has not yet been tested and thus cannot be trusted [21].

We present the design and implementation of ZEUS-a practical framework for automatic formal verification of smart contracts using abstract interpretation and symbolic model checking. ZEUS takes as input the smart contracts written in high-level languages and leverages user assistance to help generate the correctness and/or fairness criteria in a XACMLstyled template [51]. It translates these contracts and the policy specification into a low-level intermediate representation (IR), such as LLVM bitcode [66], encoding the execution semantics to correctly reason about the contract behavior. It then performs static analysis atop the IR to determine the points at which the verification predicates (as specified in the policy) must be asserted. Finally, ZEUS feeds the modified IR to a verification engine that leverages constrained horn clauses (CHCs) [55], [61], [69] to quickly ascertain the safety of the smart contract.

ZEUs leverages three key observations to be both sound and scalable. First, while the blockchain has execution akin to a concurrent system with task-based semantics, a transaction comprises of just one call chain starting from a publicly visible function in the smart contract. This observation helps significantly reduce the state space exploration for verifying most properties. Also, data dependence across transactions, such as read/write hazards among persistent state variables, requires analyzing $O\left(n^{2}\right)$ pairs of transaction interleavings. Second, smart contracts are both control- and data-driven. Thus, modeling contracts using abstract interpretation along with symbolic model checking allows ZEUS to soundly reason about program behavior. Abstract interpretation computes loop and function summaries over data domains, which are then used during the model checking phase that now operates upon a reduced state space. Lastly, $\mathrm{CHCs}$ provide a suitable mechanism to represent verification conditions, which can be discharged efficiently by SMT solvers.

ZEUS also benefits greatly from verification atop LLVM bitcode. Not only does this allow ZEUS to leverage an industry strength tool-chain for analysis, it enables ZEUS to plug in any verifier that operates upon the standardized (and formally verified [74]) LLVM bitcode. Use of LLVM bitcode also helps ZEUS to support verification of smart contracts for different blockchain platforms, including Ethereum [13] and Hyperledger Fabric [24] (or Fabric), written in diverse high-level languages, such as $\mathrm{C \# ,} \mathrm{Go} \mathrm{and} \mathrm{JAVA.} \mathrm{Note} \mathrm{that}$ most high-level languages have mature source code to LLVM bitcode translators already available. We leverage LLVM's rich API set to develop the first Solidity to LLVM bitcode translator, which faithfully implements execution semantics for majority of the Solidity syntax for verification. Furthermore, use of LLVM passes allow ZEUS to separate translation from implementation of verification checks.
This paper makes the following contributions:

(1) We classify several new and previously known issues but unstudied in the context of smart contracts and show that they can potentially lead to loss of money ( $\S$ III).

(2) We present a formal abstraction of Solidity's execution semantics for verifying smart contracts using a combination of abstract interpretation and symbolic model checking ( $\S$ IV). (3) We present the design and implementation of ZEUS ( $\S \mathrm{IV}$, and $\S \mathrm{V}$ ), a symbolic model checking framework for verification of correctness and fairness policies. We build the first Solidity to LLVM bitcode translator and provide a program analysis module that automatically inserts verification conditions given a policy specification. We also provide abstraction strategies to correctly model Solidity's execution semantics to ensure soundness. Further, we build an interactive predicate extraction tool to make it easy to specify policies for multi-party interactions in smart contracts.

(4) We present the first large scale source code analysis of Solidity-based smart contracts. Our evaluation ( $($ VI) with 22, 493 Solidity smart contracts (of which 1524 were unique) indicates that about $94.6 \%$ of them (with a net worth of over $\$ 0.5$ billion) are vulnerable to one or more correctness issues. However, we do not investigate the practical exploitability of these bugs. Additionally, we selected several representative contracts and applied contract-specific fairness criteria.

(5) ZEUS is sound (with zero false negatives) and outperforms Oyente for contracts in our data set, with low false positive rate and an order of magnitude improvement in time for analysis. (6) We show ZEUS's generic applicability by leveraging it to verify smart contracts for the Fabric blockchain. We also demonstrate the ease of applying ZEUS to a verifier of choice by using SMACK [72] for verification ( $\S \mathrm{VI}-\mathrm{D})$.

\section{BACKGROUND}

Blockchain is a distributed, shared ledger that records transactions between multiple, often mutually distrusting parties in a verifiable and permanent way. These transactions are maintained in a continuously growing list of ordered "blocks", which are tamper-proof and support non-repudiation. Apart from the array of transactions, each block also includes state metadata, including the creation timestamp, the Merkle hash of the transactions, the hash of the previous block in the chain, and smart contract code and data. Mining is the process of distributed, computational review performed on each block, enabling consensus in a mutually distrusting environment.

Permissionless blockChain. Permissionless platforms allow anyone to join the network and participate in the process of block verification to create consensus. Examples of permissionless blockchain platforms include Bitcoin and Ethereum, where any miner can join the network and start mining. Permissionless platforms use consensus mechanisms, such as proof-of-work or proof-of-stake, to build trust and validate transactions. Since the permissionless blockchain is decentralized, anonymous and equally accessible to anyone, the ability to create trust and the ability to scale is low, resulting in low network throughput.

Permissioned BLOCKCHAin. A permissioned blockchain platform, such as Fabric, restricts the participants who can contribute to the consensus of the system state. In other 

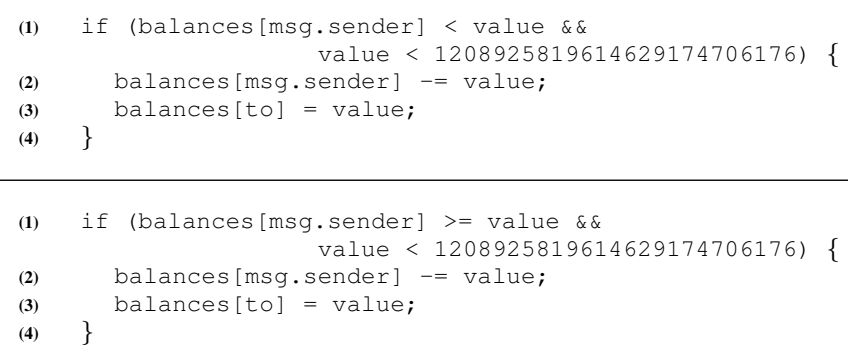

Fig. 9: Logic error in contracts [4] and [28].

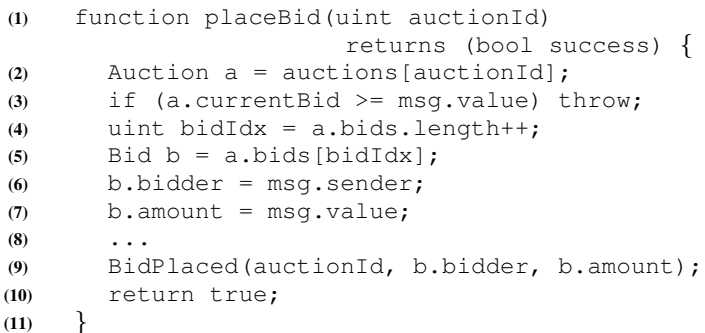

Fig. 10: An unfair auction house contract (adapted from [2]).

- A recent attack [34] on the popular MultiSig wallet contract allowed an attacker to change the owner of the wallet by invoking the initwallet function in the context of the previous owner, which did not check for double initialization and was inadvertently made a public function. The attackers were able to get away with over $\$ 30 \mathrm{mn}$ worth of Ether.

- Consider Fig. 9 that shows the exact same snippet in two contracts [4] and [28]. However, the check to determine that balance must be greater than value to allow the transfer is incorrect in the first one and correct in the other one.

(IV) Logically CORRect but UNFaIR. Consider the placeBid function from an auction house contract [2] in Fig. 10. By law, an auction in the U.S can be "with reserve" or "without reserve". If a seller is allowed to bid, the auction is "with reserve", which can affect the participants' willingness (since the seller can artificially bid up the price). Further, the seller may withdraw the property from the auction anytime prior to it being sold. However, most importantly, at such "with reserve" auctions, the seller may bid only if that right is disclosed to the participants. This contract does not disclose whether it is "with reserve" or not, and the knowledge is gleaned only by analyzing the source code. The placeBid function places no restriction on bidders willing to bid, indicating that sellers can also participate. A careful code analysis reveals that the seller can indeed withdraw the item before being sold. However, unsuspecting bidders, having no expertise in analyzing code, may lose money due to artificially increased bids or forfeit their participation fee. This contract is thus unfair to participants, and indicates the subtleties involved in multi-party interactions, where fairness is subjective.

\section{Miner's Influence}

A miner in a permissionless blockchain can order the transactions from his pool. A malicious miner can re-order transactions (while being adversarial to some participants) and obtain profit by prioritizing his own transactions.

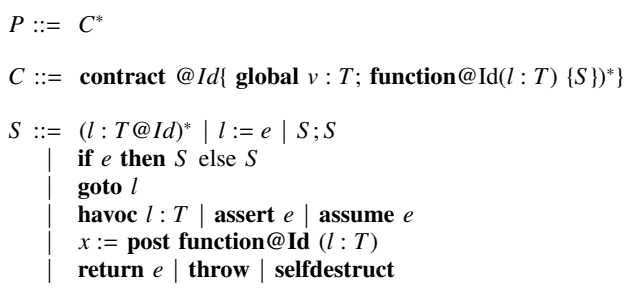

Fig. 11: An abstract language modeling Solidity.

(I) Block STATE DePendenCE. Solidity defines several block state variables, such as timestamp, coinbase, number, difficulty and gaslimit, which can be used to generate randomness [48]. All these variables are determined from the block header, and are thus, in principle, vulnerable to tampering by the block miner, who can insert suitable values to favor payouts intended for him, albeit with varying degrees of success [14]. While prior work [68] considers only timestamp, other block state variables can also lead to different Ether flows along different program paths.

(II) Transaction ORder DePendence. Concurrent systems have for long grappled with the problem of data races due to transaction ordering. While Solidity does not support concurrency, a miner can influence the outcome of a transaction due to its own reordering criteria. Since this dependence on transaction ordering is a universal blockchain feature, we consider it a limitation rather than a bug.

\section{ZEUS}

ZEUS's tool chain for smart contract verification consists of (a) policy builder, (b) source code translator, and (c) verifier. Specifically, ZEUS takes as input a smart contract and a policy (written in a specification language) against which the smart contract must be verified. It performs static analysis atop the smart contract code and inserts the policy predicates as assert statements at correct program points. ZEUS then leverages its source code translator to faithfully convert the smart contract embedded with policy assertions to LLVM bitcode. Finally, ZEUS invokes its verifier to determine assertion violations, which are indicative of policy violations.

We now present a formal overview of ZEUS's workflow and present proofs of its soundness. While we focus on Soliditybased smart contracts, ZEUS's design is generic and applicable to contracts written in any source language. Note that our formalism is inspired from [68] to maintain readability.

\section{A. Formalizing Solidity Semantics}

We define an abstract language that captures relevant constructs of Solidity programs (see Fig. 11). A program consists of a sequence of contract declarations. Each contract is abstractly viewed as a sequence of one or more method definitions in addition to declaration and initialization of persistent storage private to a contract, denoted by the keyword global. A contract is uniquely identified by $I d$, where $I d$ belongs to a set of identifiers. This invocation of the contract's publicly visible methods is viewed as a transaction.

For simplicity, we have methods with a single input variable of type $T$ (where $\operatorname{Dom}(T) \subseteq \mathbb{N}$ ) and a single variable 
that is global to the contract's functions ${ }^{5}$. Since $T$ is generic, it can represent collections and structs as well. Method invocations in Solidity can be of three types: internal, external and call. Internal and external invocations are modeled via the goto instruction or are inlined, while the call invocation is modeled separately as post. The body of a contract method is inductively defined by $S$. In contrast, the post statement can be invoked with arguments across contracts. Hence, argument $l$ (of type $T$ ) is part of post.

The semantics of our language abstract concrete values and operations. Thus, enumeration of $T$ or particular expression language $e$ remains unspecified for us. Note that the details of the expression language are not important; one can assume linear arithmetic expressions defined for any traditional imperative language. The statement havoc assigns a nondeterministic value to the variable $l$. An assert statement introduces a check of truth value of predicates in the symbolic encoding. An assume statement blocks until the supplied expression becomes true and specifies a data state at a given control location in a contract.

While a formal argument about the semantic equivalence of Solidity and our abstract language is desirable and can be established by defining abstraction functions from Solidity constructs to constructs in our abstract language, we omit it in the interest of space. Instead, we intuitively reason about the various Solidity constructs and their equivalent modeling in our abstract language. Constructs such as class, library and interfaces can be desugared as a collection of global variables and functions in our abstract language. Even compilers model them similarly when translating $\mathrm{C} / \mathrm{C}++$ code to LLVM bitcode. struct, mapping, arrays and bytes are mapped to globals. Built-in methods such as sha256 that affect the state of the same contract are modeled as external functions. Functions that operate upon addresses such as send, transfer, and call family of instructions are modeled via the post statement. Special constructs like selfdestruct are natively modeled in our abstract language. All control structures including function modifiers in Solidity can be desugared into if-then-else and goto. Solidity-style exception handling using assert, require and throw also maps directly to our abstract language using if-then and throw. Note that assert and assume in our language are used for verification, and assert has semantics different from those in Solidity. Other compiler directives such as constant and storage are also desugared, and are thus not modeled explicitly in our abstract language.

LANGUAGE SEMANTICS. The blockchain state is defined by the tuple: $\langle\langle\mathcal{T}, \sigma\rangle, B C\rangle$ where $\langle\mathcal{T}, \sigma\rangle$ is the block $B$ being currently mined. $B C$ is the list of committed blocks, and $\mathcal{T}$ denotes the multiset of completed transactions that are not yet committed. Let Vals $\subseteq \mathbb{N}$ be the set of values that expressions can take after evaluations. $\sigma$ is the global state denoted by the function $\sigma: I d \rightarrow g$ that maps contract identifiers to a valuation of the global variables, where $g \in$ Vals. Note that $\sigma$ is the state of the system reached after executing $\mathcal{T}$ in an order as specified by the miner. Finally, each miner will add $B$ to their respective copies of blockchain once it is validated.

A transaction is defined as a stack of frames represented

\footnotetext{
${ }^{5}$ We lose no generality with single local and global variables
}

by $\gamma$. Each frame is further defined as: $f:=\langle\ell, i d, M, p c, v\rangle$, where $\ell \in$ Vals is the valuation of the method-local variables $l, M$ is the code of the contract with the identifier $i d, p c$ is the program counter, and $v:=\langle i, o\rangle$ is an auxiliary memory for storing input and output. The top frame of $\gamma$ is the frame under active execution and is relevant to the currently executing transaction; it is not part of the persistent blockchain state. An empty frame is denoted by $\epsilon$. A configuration $c$, defined as $c:=\langle\gamma, \sigma\rangle$, captures the state of transaction execution and $\rightsquigarrow$ denotes the small-step operational semantics.

Table 1 lists relevant semantic rules that govern changes in the configuration. Rules for remaining sequential statements are standard. The symbol $\rightarrow$ is overloaded to illustrate a transition relation for globals and blockchain states. The symbol $\leftarrow$ indicates an assignment to an lvar.

\section{B. Formalizing the Policy Language}

Assume PVars to be the set of program variables, Func to be a set of function names in a contract (which is uniquely identified by $I d$ as defined in $\S$ IV-A) and Expr to be the set of conditional expressions specified as quantifier-free first order logic (FOL) formulae. Policy specification must use these syntactic symbols to avoid any ambiguity during verification.

ZEUS leverages user assistance to build a XACMLstyled five tuple policy specification [51] consisting of $\langle S u b, O b j, O p$, Cond, Res $\rangle$. Subject $S u b \in$ PVars is the set of source variables (one or more) that need to be tracked. Object $O b j \in$ PVars is the set of variables representing entities with which the subject interacts. Operation $O p$ is the set of side-affecting invocations that capture the effects of interaction between the subject and the object. $O p$ also specifies a trigger attribute, either 'pre' or 'post', indicating whether the predicates should hold before or after the specified operation. In other words, $O p:=\langle f$, trig $\rangle$ where $f \in F$ unc and trig $\in\{$ pre,post $\}$. Condition Cond $\in$ Expr is the set of predicates that govern this interaction leading to the operation. Finally, $\operatorname{Res} \in\{T, F\}$ indicates whether the interaction between the subject and operation as governed by the predicates is permitted or constitutes a violation.

Translation of Policy to Assertions. Our abstract language includes assertions for defining state reachability properties on the smart contract. ZEUS leverages the policy tuple to extract: (a) predicate (i.e., Cond) to be asserted, and (b) the correct control location for inserting the as sert statements in the program source.

Notice that Cond is an expression in our abstract language. Thus, taking this expression predicate and wrapping it under assert (exp) creates a statement in our abstract language. Res indicates whether the condition will appear in its normal or negated form in the assert statement. $O p$ indicates the function invocations where the predicates (as indicated by the condition) must satisfy trig. This trig attribute along with $S u b$ and $O b j$ precisely discriminates which invocations of the operation should be prefixed or suffixed with the condition. In other words, we precisely know the control locations in the abstract program $P$ where $C$ ond must be asserted.

More formally, $f: S u b \times O b j \times O p \rightarrow L o c$ where $L o c$ is the set of program locations. Operationally, this function is 


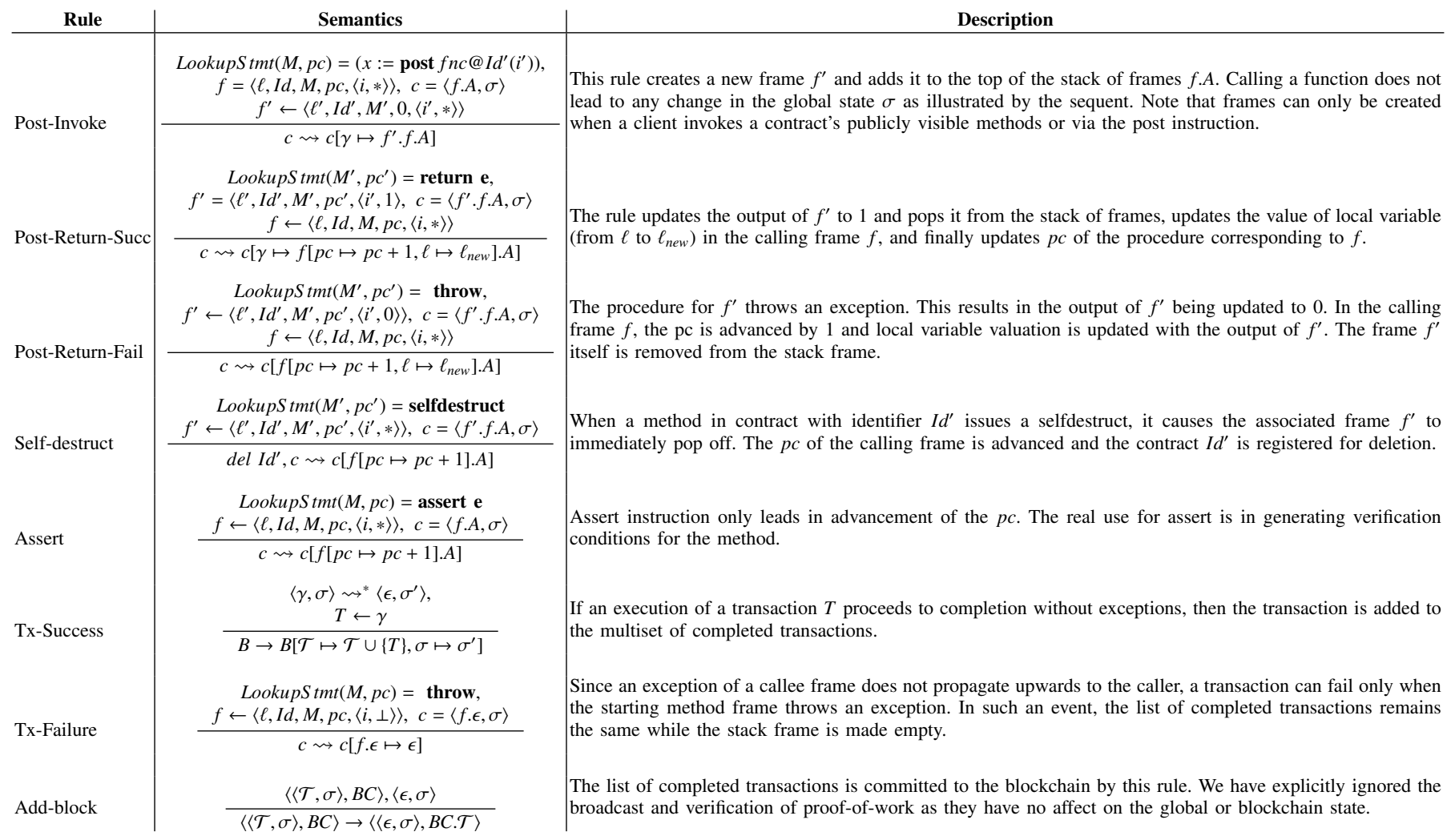

Table 1: Semantic rules for abstract assertion language. Note that $M$ can be obtained by calling LookupCode(Id, $\sigma)$ and the statement about to be executed can be obtained by the function $\operatorname{LookupStmt}(M, p c)$ where $p c$ is pointing to the next statement to be executed.

realized by performing a taint-analysis to determine the set of locations where $S u b$ and $O b j$ are conjunctively used. This set is further refined by choosing only those control locations where the specified operation is invoked. The final locations are the ones where Cond must be asserted based on trig.

\section{Soundness}

The proof for soundness of the translation from a Solidity contract to our abstract language with assertions (corresponding to policy predicates), and finally into LLVM bitcode entails the following steps. First, we discuss that translation of Solidity code into our abstract language does not affect semantic behavior. Second, we argue that a conservative placement of asserts does not affect the soundness of the approach. Third, we reduce the problem of policy confirmation to a state reachability problem. Fourth, we provide a definition of state reachability in the context of a Solidity program. Fifth, we demonstrate that by ensuring state reachability on an overapproximate version of the program, we do not miss on any program behaviors. Lastly, we argue that since our translation from this over-approximate Solidity program to LLVM bitcode is a faithful expression-to-expression translation, our overall soundness modulo the decision procedure is preserved.

(I) Transformation From Solidity to abSTRACT LANGUAGE. Since Solidity maps semantically to our abstract language (per $\S$ IV-A), this translation preserves the semantic behavior of the original program. While a formal argument about the semantic equivalence of Solidity and our abstract language is desirable, we omit it in the interest of space.
(II) EFFECT OF TAINT ANALYSIS ON SOUNDNESS. Note that taint-analysis, which is required to determine the locations at which to assert the predicates, is conservative. Thus, it may potentially insert asserts at multiple locations. While such extraneous asserts may introduce false positives, they do not affect false negatives. Hence, the approach is sound.

(iII) Semantic Interpretation of Policy CONFIRMation. Since the policies are restricted to quantifier-free FOL, policy confirmation can be reduced to a state reachability problem, i.e., does there exist a state reachable from the start state at which the policy does not hold? Formally, a policy $\phi$ holds on a program $P$ when $N \vDash \phi$, where $N$ is a formal representation of program $P$ as a state-transitioning finite automaton. Note that assert (exp) in the abstract language has different semantics than the assert statements in high-level languages such as C (per Table 1).

(IV) ASSERTION SAFETY IN A PROGRAM IMPLIES POLICY CONFIRMATION. Consider a program $\hat{P}$ (corresponding to a Solidity program $P$ ) in our abstract language but without any asserts or havocs. Let $B_{\hat{P}}$ be the set of behaviors of $\hat{P}$ described as $B_{\hat{P}}=\left\{s \mid \forall s_{0} \in I, s \in \operatorname{Reach}\left(s, s_{0}\right)\right\}$ where $I$ is the set of initial states and the relation $\operatorname{Reach}\left(s, s^{\prime}\right)$ is true iff $s \rightarrow^{*}$ $s^{\prime}$. Consider the translation $\hat{P} \rightarrow \hat{P}^{\prime}$, where $\hat{P}^{\prime}$ has asserts inserted according to the rules governed by the compilation of policies into asserts as described above.

Lemma 1: Assertion safety in $\hat{P}^{\prime} \Leftrightarrow$ Assertion safety in $\hat{P}$.

Proof: The semantic rule for the assert statement indicates no change in the data state of the program except the change 


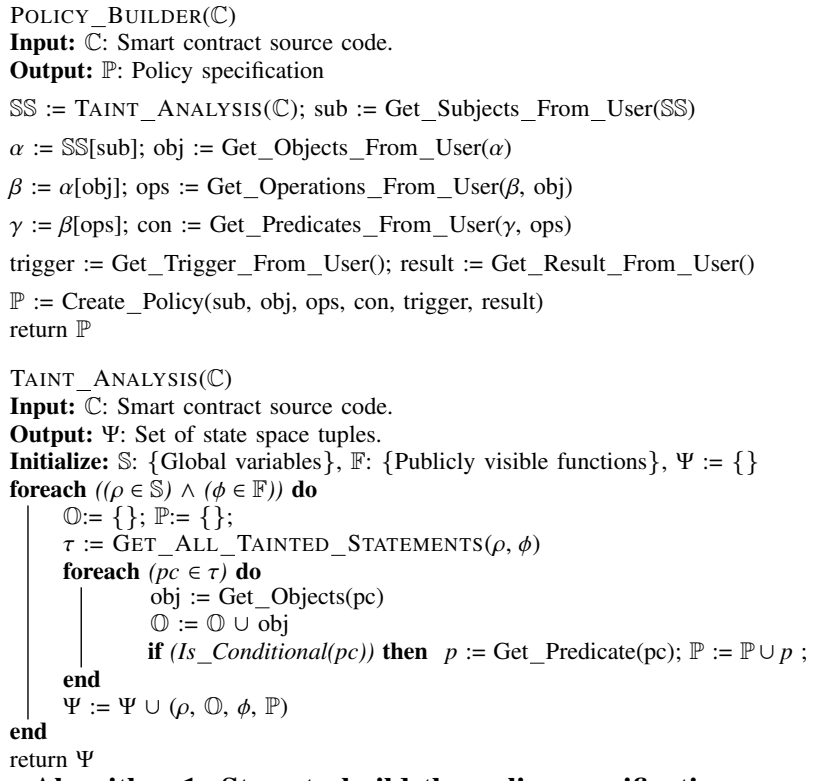

Algorithm 1: Steps to build the policy specification.

the sources and terminating at the sinks. The output of the taint analysis pass is a set of tuples consisting of the source, the objects, the sink and its corresponding path predicates. ZEUS then lists the set of all available taint sources, i.e., the globals and the environment variables, from which a user selects the subject to be tracked. It then filters the results from the taint pass that reduces the search space to tuples containing at least one of the subjects selected by the user. ZEUS then prompts the user to select the object(s), following which it further prunes the tuple list. It then displays the list of potential invocations that involve at least one or more of the subjects or objects. Upon further selection, ZEUS lists the available predicates encountered along the source to the sink. The user can compose these predicates (or specify his own) using boolean operators to form the condition in the policy, and indicate whether they are checked as a pre- or postcondition. Finally, the user indicates in the result tag whether the specification determines a violation or accepted behavior.

Fig. 13 lists the fairness criteria for the example shown in Fig. 10. Correctness polices use a similar template with the operation specifying the bug class to be detected.

\section{B. Solidity to LLVM Bitcode Translator}

ZEUS takes in a smart contract and passes it through the translator to generate its LLVM bitcode along with the debug symbol information. Subsequently, for ease of implementation, it reads the policy specification and rewrites the bitcode (instead of the Solidity source code) to inject assert conditions for the predicates as per the trigger attribute in the specification. Most Solidity statements and expressions have the same semantics as their $\mathrm{C} / \mathrm{C}++$ counterparts. We use the rich LLVM APIs to generate semantically correct bitcode while traversing the AST during code compilation (per Table 2). We handle expression translation using the standard LLVM APIs.

ENSURING SOUNDNESS. In Solidity, execution of a public function constitutes a transaction, which can be reordered at the miner. To be sound, ZEUS must correctly reason about all possible execution orders and control paths in the program.
(1) Execution order: Since there can be arbitrarily many parallel invocations of a contract's public functions with no global invocation order enforced, modeling these infinitely many execution orders is not possible. We observe that for six of the seven classes (except transaction-order dependence), bug detection is intra-transaction. In other words, the verifier needs to reason about bugs within one call chain. The ordering of transactions does not impact bug detection within a transaction. Detection of transaction-order dependence involves detecting writes and subsequent reads to sensitive global variables across a pair of transactions. Reasoning about pairs of functions suffices, because a minimal instance of transactionorder dependence must manifest across at least two function invocations. Ordering of remaining functions is immaterial for detecting the bug. Thus, for a contract with $n$ publicly available functions, ZEUS must reason about $O\left(n^{2}\right)$ possible orderings. ZEUS generates a set of main functions, which act as the entry point for verification. Specifically, a main function is a harness that havocs all the global state before invoking a publicly defined function. For transaction-order dependence, ZEUS havocs the global state and invokes pair-wise permutations of all public functions from within a main function. Further, ZEUS havocs the entire global state upon invocation of any member of the call family.

(2) Path traversal: In Solidity, global variables hold state across executions. Modeling and reasoning all such states in one static analysis execution is impractical. Thus, ZEUS abstracts the values of all globals in the contract, including the block and transaction state variables, to the entire data domain corresponding to the data type. For example, a global of type uint256 is modeled as having an integer domain, and can take values anywhere between 0 and INT_MAX. For any concretely defined starting values, ZEUS automatically havocs them to explore the entire data domain. Keeping the initial value constant does not reason about all possible executions since the value may be incremented in subsequent contract executions and may lead to potential exploitability in the future. Thus, a single static execution suffices to analyze all possible control paths.

Modeling Solidity Syntax. Zeus supports complex Solidity syntax, including inheritance, external functions, tuple expressions, modifiers, operations over nested struct definitions, iteration over maps and arrays, and memory allocation/de-allocation. We discuss a few of them below:

(1) Inheritance: Solidity allows multiple inheritance amongst the contracts. ZEUS follows the same logic used by Solidity to implement inheritance, i.e., the base contract on the extreme right in the declaration overrides all functions previously declared by other base contracts. Specifically, ZEUS generates the LLVM bitcode per contract, and then follows the said order to patch the overriding functions visible in the derived class.

(2) External functions: Solidity allows one contract to call into another contract. Furthermore, Solidity mandates that all external functions only take in primitives as input and the returns are also of primitive types [36]. While ZEUS cannot resolve such functions at compile time, it over approximates their behavior for soundness and assumes that these external functions return a non-deterministic value. This non-determinism soundly models the execution semantics of these external functions, i.e., the return can take any value.

(3) Arrays: Zeus does not implement dynamic arrays in 


\begin{tabular}{|c|c|c|c|}
\hline AST Node & Abstract & LLVM API & Comments \\
\hline ContractDefinition & contract@Id $\{\ldots\}$ & Module & $\begin{array}{l}\text { Creates a new module, sets the data layout, generates the definition of global } \\
\text { variables and functions, and writes a main function which serves as the driver. }\end{array}$ \\
\hline EventDefinition & function@Id(1:T) $\{\mathrm{S}\}$ & $\begin{array}{l}\text { FunctionType, } \\
\text { Function }\end{array}$ & $\begin{array}{l}\text { Creates a new function with the return type void, the arguments type as specified } \\
\text { in the event, and inserts it in the given module. }\end{array}$ \\
\hline FunctionDefinition & function@Id(1:T) $\{\mathrm{S}\}$ & $\begin{array}{l}\text { FunctionType, } \\
\text { Function }\end{array}$ & Creates a new function with the given return type, arguments and the body. \\
\hline Block & $\{\mathrm{S}\}$ & BasicBlock & $\begin{array}{l}\text { Creates a BasicBlock inside the LLVM IR, sets the insertion point to this block, } \\
\text { and iterates over the statements to generate the IR for each one. }\end{array}$ \\
\hline VariableDeclarationstatement & $(1: T)^{*}$ & $\begin{array}{l}\text { CreateStore, } \\
\text { CreateExtorTrunc }\end{array}$ & $\begin{array}{l}\text { Iterates over all the variables declared in this statement, allocates the variable } \\
\text { using the VariableDeclaration node, and stores the initial value (or a default } \\
\text { value) to the allocated space. (after sign / zero extension / truncation if needed). }\end{array}$ \\
\hline VariableDeclaration & $(1: T)$ & $\begin{array}{l}\text { GlobalVariable, } \\
\text { CreateAlloca }\end{array}$ & $\begin{array}{l}\text { For a global variable, uses the GlobalVariable API to define a global variable, } \\
\text { otherwise, allocates space using the alloca instruction. }\end{array}$ \\
\hline Literal & $\ell$ & ConstantInt & Allocates a constant value for the various types of integers of varying widths. \\
\hline Return & return e & $\begin{array}{l}\text { ReturnInst, } \\
\text { CreateExtOrTrunc, } \\
\text { CreateGEP }\end{array}$ & $\begin{array}{l}\text { Uses other AST nodes to generate the expression to return, dereferences it, } \\
\text { extends / truncates the value, and returns the value using the return instruction. }\end{array}$ \\
\hline Assignment & $1:=\mathrm{e}$ & $\begin{array}{l}\text { CreateExtractValue, } \\
\text { CreateExtorTrunc, } \\
\text { CreateLoad, } \\
\text { CreateStore, } \\
\text { CreateBinop }\end{array}$ & $\begin{array}{l}\text { Generates the right hand side of the expression. A tuple is unpacked. For } \\
\text { compound assignments, the corresponding binop instruction is created, and the } \\
\text { result is sign/zeroextended/truncated and stored in the left hand side. }\end{array}$ \\
\hline Expressionstatement & $\mathrm{e}$ & & Calls the ASTNode for the Expression to generate the LLVM IR. \\
\hline Identifier & Id & $\begin{array}{l}\text { ValueSymbolTable, } \\
\text { GlobalVariable, } \\
\text { getFunction }\end{array}$ & $\begin{array}{l}\text { Checks for the identifier inside the local variables, global variables or the } \\
\text { functions written in the contract, and returns the appropriate LLVM object. }\end{array}$ \\
\hline IfStatement & if e then $\mathrm{S}$ else $\mathrm{S}$ & $\begin{array}{l}\text { BasicBlock, } \\
\text { CreateBr, } \\
\text { CreateCondBr } \\
\end{array}$ & $\begin{array}{l}\text { Generates the condition variable inside the current block, creates a conditional } \\
\text { branch, and branches to either the true or the false branch. In the absence of the } \\
\text { false branch, uses the branch instruction to fall through. }\end{array}$ \\
\hline Functioncall & goto or post & $\begin{array}{l}\text { CreateExtOrTrunc, } \\
\text { Createcall, } \\
\text { Function }\end{array}$ & $\begin{array}{l}\text { Generates the arguments for the function call, fixes their type according to the } \\
\text { solidity semantics, and creates a call to the required function. }\end{array}$ \\
\hline Whilestatement / Forstatement & $\begin{array}{l}\text { if e then goto } 1 \\
\text { else } \mathrm{S}\end{array}$ & $\begin{array}{l}\text { BasicBlock, } \\
\text { CreateCondBr }\end{array}$ & Generates the conditional variable, the body of the loop, and a branch instruction. \\
\hline StructDefintion & $\mathrm{T}$ & Struct Type & Generates a structure of the same type as in the solidity contract. \\
\hline Throw & throw & $\begin{array}{l}\text { Function, } \\
\text { Createcall }\end{array}$ & Calls the system's exit function. \\
\hline Break / Continue & if e then gotol & CreateBr & a stack of the break/continue tags and branches to the appropriate label. \\
\hline
\end{tabular}

Table 2: Expression to expression translation from Solidity code to LLVM bitcode.

LLVM bitcode but uses a static array with large length. This minor tweak preserves the semantic meaning of the contracts and makes them amenable for verification. All strings and byte accesses are also modeled as arrays with integer domain.

(4) Rational Ether: Solidity does not implement floating point arithmetic. It instead uses rational numbers to implement fractional payouts. ZEUS converts such rational payments into lower monetary units to allow integer arithmetic in LLVM bitcode. For example, ZEus converts $1 / 4$ ether to 250 finney.

HANDLING LLVM OPTIMIZATIONS. LLVM's optimizer can run aggressive passes eliminating any non-side affecting variables and function calls. However, this can adversely impact the verification result. For example, if return values from invocations such as send are not used, both the send call and the return value are optimized out. This optimization causes problems in detecting scenarios described earlier in $\S$ III-A and $\S$ III-C. Further, the verifiers may invoke their own optimization passes that may mess with the LLVM bitcode translation from Solidity code. In an effort to remain faithful to the semantics envisioned by the contract writer, ZEUS creates a global variable for each external function return value and enforces no optimization on all functions.

LiMiTaTions. Our prototype of ZeUs has a few limitations across policy specification, translation and verification.

(1) Fairness properties involving mathematical formulae are harder to check. For example, $25 \%$ can be represented in several different forms. ZEUS depends on the user to accurately define policies that involve such mathematical representations. (2) ZEUS is faithful to most Solidity syntax. However, constructs like throw and selfdestruct which have no exact LLVM bitcode transformation are modeled as a program exit. Further, runtime EVM parameters such as gas consumption cannot be precisely computed at the source level. Thus, Zeus is overly conservative in its runtime behavior modeling and does not explicitly account for these parameters. (3) ZEUS does not support virtual functions in contract hierarchy, i.e., use of super keyword, which resolves the function call at runtime, dependent on the final inheritance graph. We manually analyzed the 23 such contracts in our dataset and resolved the super linkages.

(4) Solidity's assembly block allows use of EVM bytecode alongside regular Solidity statements. Even though real-world contracts rarely use assembly (only 45 out of 22,493 contracts in our data set use it), ZEUS is conservative and does not analyze contracts with an assembly block.

(5) ZEUS supports verification of safety properties, i.e., state reachability expressible via quantifier-free logic with integer linear arithmetic. Verification of liveness (i.e., something good must eventually happen) requires support for linear temporal logic, and is currently not supported by ZEUS. Extending ZEUS to support other kinds of properties such as trace- or hyperproperties does not require changes to the core design and we leave it for future work. 


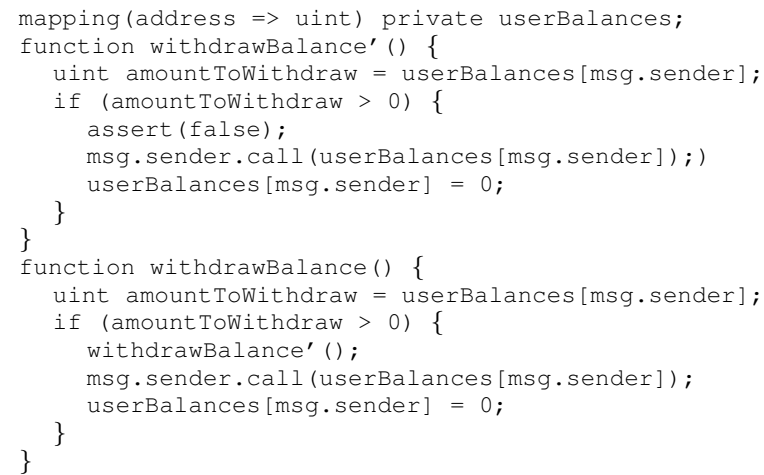

Fig. 14: Same-function reentrancy detection for example in Fig. 2.

\section{Handling Correctness Bugs}

ZEUS provides verification for the correctness issues described in $\oint$ III-A and $\S$ III-C. We discuss the verification logic for them as implemented in several LLVM passes below.

(1) Reentrancy: Reentrancy in Solidity can happen via the call method. send only invokes the default function with limited gas for logging purposes. ZEUS handles same-function reentrancy by first cloning the function under consideration, and inserting a call to the clone before the invocation to call. Fig. 14 shows the patched function for the example in Fig. 2. Note that ZEUS ensures that the patch is done within the same basic block so as to ensure that if the cloned function is called, then the invocation to call is also made. Further, we also assert false before the call code. If the verifier finds a path leading to this assert, it indicates a bug.

Cross-function reentrancy can be handled similarly by patching different functions. However, it is not scalable due to state space explosion even with small number of functions. We leave efficient detection of cross-function reentrancy for the future.

(2) Unchecked send: Detection of unchecked send bug requires identifying any subsequent accesses to global state variables in case of a failed send call. ZEUS initializes a global variable checksend to true and takes its conjunction with return value from every send operation. For every subsequent write of a global variable, an LLVM pass automatically inserts an assert (checksend) in the bitcode.

(3) Failed send: Recall that the aim here is to prevent reverting the transaction by not invoking throw on a failed send call. The detection of this bug leverages the same check as the unchecked send scenario, but places the assertion ahead of the throw. While the throw encountered may be due to some other condition in the code, the counterexample indicates a possibility of reverting the transaction due to control flow reaching a throw on a failed send, not necessarily the immediate throw associated with the send.

(4) Integer overflow/underflow: An LLVM pass implements the overflow/underflow detection checks for all arithmetic operations [20], consistent with Solidity's semantics, i.e., an overflow/underflow for integers causes a wrap around.

(5) Block/ Transaction state dependence: Bug detection for these classes requires context sensitive information. For example, block state dependence requires determining if block variables, such as timestamp, flow into send or call. We implement our own taint analysis pass over LLVM bitcode and use symbolic model checking to eliminate infeasible paths.

\begin{tabular}{l|r|r|r|r|r} 
& & \multicolumn{2}{|c|}{ \#LOC $(\mathrm{K})$} & \#Send/Call & \#Ext. Calls \\
\hline DAO & \#Contracts & Source & LLVM & 252 & 350 \\
Game & 140 & 2.8 & 24.3 & 851 & 16 \\
Token & 244 & 23.3 & 609.2 & 311 & 271 \\
Wallet & 290 & 25.2 & 385.9 & 186 & 6 \\
Misc. & 72 & 10.8 & 105.9 & 1102 & 498 \\
\hline Total & 778 & 47.6 & 924.3 & 2702 & 1141
\end{tabular}

Table 3: Characterization of the dataset.

Note that Solidity's lack of pointer arithmetic, unlike $\mathrm{C} / \mathrm{C}++$, eases our taint tracking implementation.

(6) Transaction order dependence: We detect transaction order dependence by determining potential read-write hazards for global variables that can influence Ether flows. Specifically, we taint all global variables that are written to and then determine if this taint flows into a send or call.

\section{EVALUATION}

EXPERIMENTAL SETUP. All experiments were performed atop an IBM System x3850 X5 machine having 4 Intel Xeon E7-4860 CPUs at $2.27 \mathrm{GHz}$ with 10 cores each and 2 threads/core, and 512 GB of RAM, running 64-bit Ubuntu v14.04. We built our Solidity to LLVM bitcode translator over solc [41], which is compatible with LLVM 3.6. We used a stable build of Seahorn [37] (snapshot of March 31 ${ }^{s t}$ ) as our verifier and set a timeout threshold of 1 minute. For comparisons with Oyente, we use their snapshot as available on April $15^{\text {th }}$, and keep a timeout duration of 30 minutes [68].

\section{A. Data Set}

We periodically scraped Etherscan [18], [19], Etherchain [12] and EtherCamp [11] explorers over a period of three months and obtained source code for 22, 493 contracts at distinct addresses. We discounted 45 contracts that had an assembly block in their source, and obtained 1524 unique contracts (as per their sha256 checksum). In the remainder of the section, we present results only for these unique contracts, unless specified otherwise.

We analyzed all unique contracts and classified them under five categories. "DAO" enlists all contracts that involve DAO-style investment. Contracts involving games and decentralized gambling, are clubbed under "Game". "Token" contracts implement the standard tokens for designing financial instruments. "Wallet" contracts implement a user wallet. All other contracts are listed under "Misc". Table 3 summarizes their characteristics. We note that contracts in the "DAO" and "Token" categories leverage a lot of external functionality, unlike "Game" and "Wallet" that appear to be self-contained. "Game" contracts involve significantly more number of send/call invocations than any other category, indicating a lot of Ether transfer between participants. "Misc." contracts account for half of the unique contracts, indicating the diverse nature of contracts available on Ethereum. Lastly, the generated LLVM bitcode is an order of magnitude more than the source LOC, since it is unoptimized.

Fig. 15a shows the frequency of duplicates across our data set. We observe that less than $5 \%$ contracts have more than 10 duplicates. For example, one wallet contract [49] in our data set was duplicated $10.3 \mathrm{~K}$ times. Further, the frequency of 


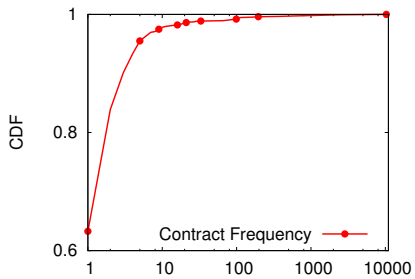

(a) Frequency of duplicate contracts.

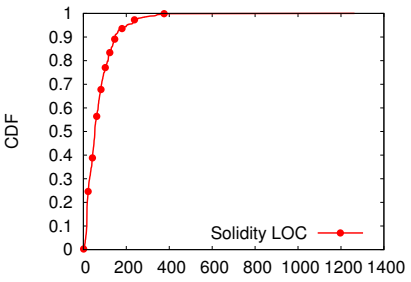

(b) Source LOC across contracts.

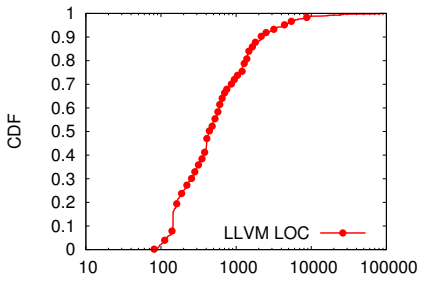

(c) LLVM LOC across contracts.

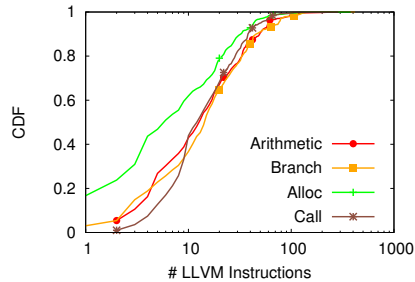

(d) Frequently used LLVM operations.

Fig. 15: Data set characterization.

duplicate token contracts at different addresses was also high. In other words, contracts providing useful functionality are more likely to be duplicated. Fig. 15b plots the source LOC in Solidity for the unique contracts. The total source LOC was over $111 \mathrm{~K}$, while mean and median were 74 and 54 LOC respectively. The largest contract we analyzed had 1405 LOC. However, over $90 \%$ of the contracts have 200 LOC or less.

ZEUS generates more than $1 \mathrm{~K}$ LOC of LLVM bitcode for around $30 \%$ of the contracts, with maximum being 91,338. (per Fig. 15c) The mean and median bitcode per contract were 1354 and 439 respectively. In all, ZEUS verified over $2 \mathrm{M}$ lines of bitcode. Note that our LOC measurements did not include any blank lines or comments. Lastly, we plot the frequently occurring operations in the bitcode. Fig. 15d presents the results for top four classes of operations, besides memory load, store and GetElementPtr. We observe that "arithmetic" operations are as frequent as "comparison", "call" and "alloc" operations.

\section{B. Results with Solidity-based Smart Contracts}

Correctness AND Miner's Influence. We evaluate ZEUS for all 1524 unique contracts for issues described in $\S$ III-A and $\S$ III-C, and compare with Oyente for the common bug classes, i.e., reentrancy, unchecked send, blockstate dependence and transaction-order dependence. We note output for each scenario as either "Safe" or "Unsafe" (i.e., there is a potential issue). In case the verifier throws an error or quits before the timeout, we categorize the contract as "No Result". All other cases are categorized under "Timeout".

Note that Solidity contracts are small and have few local/global variables, which makes it tractable to capture/track dependencies. Further, since no tools exist to ascertain the ground truth, we manually validated each result to determine the set of false positives and negatives. A false positive occurs when ZEUS returns "Unsafe" but the contract is actually "Safe", while a false negative occurs when ZEUS returns "Safe" when the contract is actually "Unsafe". The false alarm rate is the ratio of false positives over the total results returned, i.e., both "Safe" and "Unsafe". Table 4 lists the results for both ZEUS and Oyente. The entire set of results are also available at https://goo.gl/kFNHy3.

(1) Reentrancy: ZeUS detects 54 contracts as vulnerable to reentrancy. More importantly, it gives 0 false positives and negatives, primarily due to its callee function patching mechanism (per $\S \mathrm{V}-\mathrm{C}$ ). In contrast, Oyente reports 265 contracts to be susceptible to reentrancy. Since Oyente does not distinguish between send and call functions at the bytecode level, it also considers reentrancy on send, which cannot occur (recall $\S$ III-A). Hence, it reports a large number of unsafe contracts, with a high false alarm rate of over $31 \%$.

(2) Unchecked send: ZEUS reports 324 contracts affected by the unchecked send bug, with 3 false positives and 0 false negatives. We analyzed the offending contracts and observed that ZEUS's over-approximation in havocing all globals to traverse control flow not intended by the contract developer and detect bugs along those paths. In contrast, Oyente marked 112 contracts as unsafe with no result in 203 contracts. Further, it reports a high false alarm rate of around $7.5 \%$.

(3) Failed send: ZEUS detects 447 contracts vulnerable to the failed send bug with 0 false positives and negatives.

(4) Integer overflow/underflow: Smart contracts involve a lot of arithmetic operations (per Fig. 15d), and contract writers typically do not check for overflow/underflow conditions. This is corroborated by the fact that 1095 of the 1524 contracts (or around $72 \%$ ) are vulnerable to this bug.

However, ZEUS also reports 40 false positives (i.e., a false alarm rate of $2.7 \%$ ). We manually analyzed all false positives and observed that they stem due to ZEUS havocing all globals. For example, in one contract the percentage payout was declared a global with a fixed value. However, ZEUS initializes it to the entire data domain, following which an operation using the payout variable causes the operation to overflow. A better program analysis (or abstract refinement) of smart contracts can help weed out such cases, where it is not required to assign the entire data domain to a global variable. (5) Transaction state dependence: ZEUS found 1513 contracts out of 1524 to be safe, while only 8 contracts were deemed unsafe, with 0 false positives and negatives. ZEUS was successfully able to detect the vulnerability as described in Fig. 6 in the contract LittleEthereumdoubler [17].

(6) Block state dependence: ZEUS found 250 contracts to be vulnerable, with 0 false positives/negatives. In contrast, Oyente marked 15 contracts as unsafe, and failed to provide results for 711 contracts (either due to timeout or no result). ZEUS is conservative and considers all block parameters can be modified, while Oyente considers only timestamp.

We observed that solWallet [49], which has over 1.4 million Ether in balance, is also susceptible to the block state dependence bug. It uses now (an alias for block. timestamp) that can easily be tampered with by the miner [14]. For example, a miner can use a value of now, which can lie anywhere between the current timestamp and 900 seconds in the future, and allow monetary transactions (close to the end of the day) even when its daily limit has been exhausted. 


\begin{tabular}{|c|c|c|c|c|c|c|c|c|c|c|c|c|c|c|}
\hline \multirow[b]{2}{*}{ Bug } & \multicolumn{7}{|c|}{ ZEUS } & \multicolumn{7}{|c|}{ Oyente } \\
\hline & Safe & Unsafe & No Result & Timeout & False +ve & False -ve & $\begin{array}{l}\% \text { False } \\
\text { Alarms }\end{array}$ & Safe & Unsafe & No Result & Timeout & False +ve & False -ve & $\begin{array}{l}\% \text { False } \\
\text { Alarms }\end{array}$ \\
\hline Reentrancy & 1438 & 54 & 7 & 25 & 0 & 0 & 0.00 & 548 & 265 & 226 & 485 & 254 & 51 & 31.24 \\
\hline Unchkd. send & 1191 & 324 & 5 & 4 & 3 & 0 & 0.20 & 1066 & 112 & 203 & 143 & 89 & 188 & 7.56 \\
\hline Failed send & 1068 & 447 & 3 & 6 & 0 & 0 & 0.00 & & & & & & & \\
\hline Int. overflow & 378 & 1095 & 18 & 33 & 40 & 0 & 2.72 & & & & & & & \\
\hline Tx. State Dep. & 1513 & 8 & 0 & 3 & 0 & 0 & 0.00 & & & & & & & \\
\hline Blk. State Dep. & 1266 & 250 & 3 & 5 & 0 & 0 & 0.00 & 798 & 15 & 226 & 485 & 2 & 84 & 0.25 \\
\hline Tx. Order Dep. & 894 & 607 & 13 & 10 & 16 & 0 & 1.07 & 668 & 129 & 222 & 485 & 116 & 158 & 14.20 \\
\hline
\end{tabular}

Table 4: ZEUS's evaluation and comparison with Oyente [68].

(7) Transaction order dependence: ZEUS reported 607 contracts (or 39.8\%) as unsafe, with 16 false positives, and 0 false negatives. The false positives stem from havocing the globals leading to traversal of paths not intended by the developer. In contrast, Oyente reported 129 contracts as unsafe along with no decision for 707 contracts. It reported a false alarm rate of $14.2 \%$; an order of magnitude more than ZEUS.

SUMMARY. The above results enable four key observations:

- 21,281 out of 22,493 contracts (or 94.6\%) containing more than $\$ 0.5$ billion worth of Ether are vulnerable to one or more bugs. Across the unique contracts, 1194 out of 1524 contracts were found to be vulnerable to one or more bugs.

- ZEUS's use of abstract interpretation along with symbolic model checking for verification makes it sound. We observed zero false negatives for all the seven bug classes. The low false alarm rate can be further mitigated by improved program analysis to weed out scenarios not intended by the developer. - Use of CHCs enable quick verification, with only 44 out of 1524 contracts (or $2.89 \%$ ) timing out in at least one bug class. ZEUS's timeout threshold is fairly low at just 1 minute.

- Oyente is neither sound nor complete, and reports a high false alarm rate for three of its four bug classes. Further, it times out or gives no result for 711 contracts (or 46.7\%) in our dataset. These numbers are consistent with their published dataset, where almost half the tests gave no result [33].

Discussion. Zeus determines a contract as either safe or unsafe, i.e., if a contract is vulnerable in principle or not. An unsafe result does not guarantee a trivial exploit. For example, several contracts are vulnerable to integer overflow because they do not check for the bounds. Thus, ZEUS marks them as unsafe. Even though possible in principle, these contracts may not be susceptible to an immediate exploit, say when the payouts use uint 256 for calculation. Similarly, contracts using timestamp for control flow, may not be affected immediately, but a path may exist with a certain value of the timestamp that affects the Ether flow in a send invocation.

FAIRNESS. We select representative contracts from the four classes (per $\S$ VI-A) and apply contract specific properties along with a common fairness policy across all contracts.

(1) DAO: CrowdFundDao [9] implements a DAO scheme allowing investors to choose when to pay and withdraw their funds. We implemented two policies: (a) blacklisted developers cannot participate in the scheme, and (b) the investment must be more than a threshold limit. ZEus determined that none of these checks were encoded in the contract.

(2) Game: DiceRoll [10] is a dice game where players join a game by placing a bet. We implemented a policy that the number of dice rolls for a player must be limited. We observed that the game did not cap the number of dice rolls per user. (3) Token: Tokens, such as StandardToken [42], are used to implement financial instruments. While most of them consider integer underflow possibilities on the sender side, it is important for them to consider whether token transfer could result in overflows on the receiver. We implemented this policy on several contracts and observed that some of them, such as Campaign [5], do not consider overflows at the recipient.

(4) Wallet: Wallet [49] implements several functionalities for users, including a daily withdrawal limit. We check for two policies: (a) a user cannot send money to themselves, and (b) there is a limit on the amount being transferred per transaction. We observed that both these policies report a violation.

(5) Common policy: Solidity provides selfdestruct to kill a contract and return its balance to an address. We check if the construct is invoked only by the owner. We observed that 284 out of 1524 contracts had this construct, with around 5.6\% reporting a violation of the policy with no false alarms.

\section{Performance}

(I) InSTRuCTION OVERHEAD. Zeus's Solidity to LLVM bitcode translator introduces checks for several bugs described in $\S$ III-A and $\S$ III-C. Fig. 16a plots a CDF of this instruction overhead due to additional LLVM bitcode LOC introduced per contract. We observe that ZeUs introduces less than 50 LOC for $97 \%$ of contracts across five of the seven bug classes. For integer overflow/underflow, ZEUs's checks account for less than 200 LOC in $95 \%$ of the contracts. However, detection of transaction order dependence incurs maximum overhead, with $20 \%$ contracts requiring over 500 LOC for the required checks.

(II) Analysis Complexity. We measure the verification complexity by determining the number of rules generated and their depth per contract across the seven bug classes. Fig. 16b and Fig. 16c plot the results. We observe that $75 \%$ of contracts across all categories generate less than 50 rules with depth of around 700. Overall, integer overflow generates maximum constraint rules and depth across all categories, with a maximum of 1035 rules and depth of 277,345. This behavior is consistent with our observation that contracts have significant amount of arithmetic operations (recall Fig. 15d).

(III) Analysis Time. We determine Zeus's verification time for each unique contract in our data set and compare against Oyente. Fig. 16d plots the CDF of the results. We observe that ZeUs takes a minute or less for verifying $97 \%$ contracts (as indicated by the vertical line). Only 44 contracts out of 1524 timeout or give no result for one or more bug classes. In contrast, Oyente returns results for only $40 \%$ contracts within one minute. Furthermore, it provides no result or timeouts (even after 30 minutes) for around $43 \%$ contracts. 


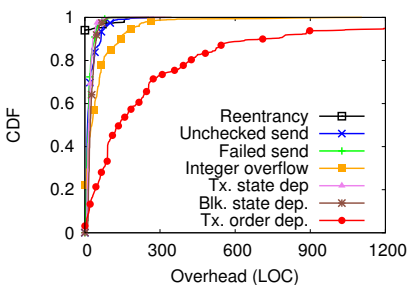

(a) LLVM bitcode instruction overhead.

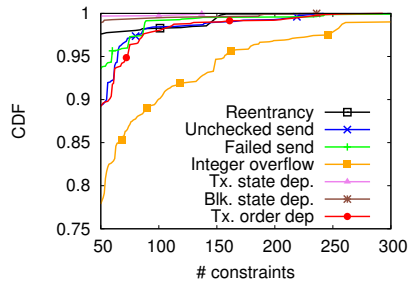

(b) Verification rules generated.

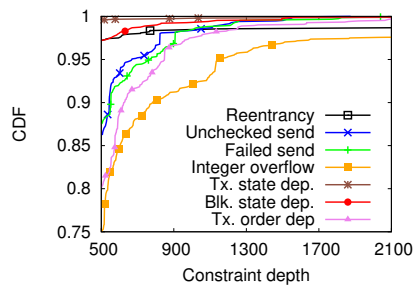

(c) Verification constraints generated.

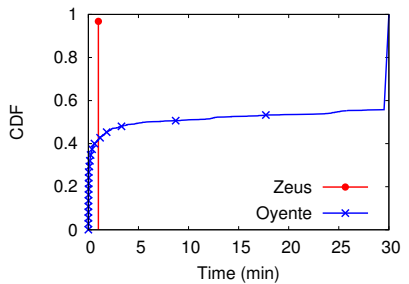

(d) Verification time in minutes.

Fig. 16: ZEUS's performance.

\section{Case Studies}

We now demonstrate ZEUS's versatility with other blockchain platforms and verification engines. First, we port a popular Solidity contract to Fabric [24]. Second, we use SMACK [72] to verify fairness policies for the said contract. We describe our experiences below.

Simple Dice [39] is a popular multi-player gambling contract, where players put in a minimum deposit (along with a fee) to play. The players have a $25 \%$ chance of winning the entire balance. Also, every 40th player wins the jackpot, which is considerably more than the balance. The fee and deposit rate can only be changed by the owner, and is publicly visible.

To validate the contract, we check the following five policies: (a) a minimum deposit is required to play the game, (b) every 40th player does win the jackpot, (c) only the owner can change the deposit and fee rate, (d) the owner cannot participate in the game, and (e) every player must have an equal chance of winning the jackpot, i.e., a player must not have multiple entries when playing for the jackpot.

(I) FABRIC. Smart contracts in Fabric can be written in high-level languages, such as Go and JAVA. Solidity's global variables that persist state across transactions are mapped on to the blockchain. In contrast, high-level languages do not have this support; the globals share state across functions calls. Fabric gets around this problem by defining a shim layer for each high-level language that exports APIs to allow smart contracts to explicitly manage state atop the blockchain.

We ported the Simple Dice contract to Go and linked it against Fabric's mock-stub [25]. However, we noticed that the mock-stub takes strings as input and converts them to the required data types using standard Go libraries. Since Seahorn does not have support for strings, we fixed the mockstub to take integers as input. While policy specification is automated for Solidity, we manually edited the Go code and placed the correct assertions along the required program paths, corresponding to the above mentioned policies. We then leveraged ligo [27] to generate LLVM bitcode for Simple Dice and verified the policies with Seahorn.

(II) SMACK. To leverage SMACK as the verifier of choice, we had to make three key modifications. First, since SMACK supports integer operations up to 64 bytes [30], we had to port our Solidity to LLVM bitcode translator to work with 64 bytes. Note that Solidity supports integers of length 256 bytes. Second, SMACK, unlike Seahorn, requires developers to use different APIs to request for the entire integer domain [40].
For example, one has to use __VERIFIER_nondet_ushort for modeling integer domains for unsigned short, and VERIFIER_nondet_uint for unsigned int. Third, the $\bar{A} \bar{P} I s$ for invocation to the verifier are different between SMACK and Seahorn. Overall, we required around 50 lines of modifications to ZEUS to make it compatible with SMACK. With our SMACK-compatible ZEUS, we verified the five fairness policies for Simple Dice as described earlier.

Our experiences with both Fabric and SMACK suggest that it is easy to extend ZEUS to other blockchain platforms and verifiers with only minor changes.

\section{RELATED WORK}

SMART CONTRACT BUG Detection. We now compare and contrast ZEUS with related work in the area of smart contract bug detection, apart from Oyente [68], Bhargavan et al. [54] and Why3 [22], [50], which we have discussed earlier. Delmolino et al. [58] document several classes of mistakes when developing contracts, suggest ways to mitigate these errors, and advocate best practices for programming smart contracts. In contrast, ZEUS presents a formal verification framework for smart contracts that enables users to build and verify correctness and fairness policies over them.

Policy SPecification. Naccio [60], PoET/Pslang [59] and Polymer [53] enable policy specification for security properties. Like prior work, ZEUS ensures that the verification policy is defined separately from the main application. This separation of logic makes it easier to understand, verify, and modify the security policy. XACML [51] defines a declarative fine-grained, attribute-based access control policy language that inspires ZEUS's syntax for policy declaration.

\section{CONCLUSION}

We present the design and implementation of ZEUS-a framework for analyzing safety properties of smart contracts. ZEUS leverages abstract interpretation and symbolic model checking, along with the power of CHCs to quickly ascertain the verification conditions. We build the first Solidity to LLVM bitcode translator to automatically insert verification conditions given a policy specification. Our evaluation with over $22.4 \mathrm{~K}$ Solidity smart contracts indicates that about $94.6 \%$ of them (with a net worth of more than $\$ 0.5$ billion) are vulnerable. ZEUS is sound (with zero false negatives) and significantly outperforms Oyente for contracts in our data set, with low false positive rate and an order of magnitude improvement in time for verification. 


\section{ACKNOWLEDGEMENT}

We thank the anonymous reviewers for their valuable comments. We are also grateful to Prasad Naldurg for his feedback on an earlier draft of the paper.

\section{REFERENCES}

[1] "Analysis of the DAO exploit," http://hackingdistributed.com/2016/06/ 18/analysis-of-the-dao-exploit/.

[2] "Auction house," https://git.io/vFAl6.

[3] "Blockchain investment in 2016," https://www.cryptocoinsnews.com/ pwc-expert-1-4-billion-invested-blockchain-2016/.

[4] "Buggy contract," https://live.ether.camp/account/ dfa42284475636ecc1e04f519b075ec7f1e04f48/contract.

[5] “CampaignToken," https://etherscan.io/address/ 0xa0388ffb2a3c198dee723135e0caa423840b375a.

[6] "Computation in Fallback Function," https://ethereum.stackexchange.com/questions/5992/how-muchcomputation-can-be-done-in-a-fallback-function.

[7] "Contest contract," https://etherscan.io/address/ 0x98086130278fe48f2f0330e330df6ed6c91ce4f7\#code.

[8] "Cross-function Race Condition," https://git.io/vFA4y.

[9] "CrowdFundDAO," https://live.ether.camp/account/ 9b37508b5f859682382d8cb6467a5c7fc5d02e9c/contract.

[10] “DiceRoll," https://ropsten.etherscan.io/address/ 0xb95bbe8ee98a21b5ef7778ec1bb5910ea843f8f7\#code.

[11] "EtherCamp," https://live.ether.camp/.

[12] "Etherchain," https://www.etherchain.org/contracts.

[13] "Ethereum," https://www.ethereum.org/.

[14] "Ethereum Block Protocol," https://git.io/vFA8I.

[15] "Ethereum Blog," https://blog.ethereum.org/2016/06/10/smart-contract-security/.

[16] "Ethereum Contract Security," https://git.io/vFA8a.

[17] "Ethereum Doubler," https://etherscan.io/address/ 0x83651a62b632c261442f396ad7202fe2a4995e3a\#code.

[18] "Etherscan," https://etherscan.io/accounts/c.

[19] "Etherscan - Ropsten," https://ropsten.etherscan.io/accounts/c.

[20] "Exception on overflow \#796," https://git.io/vFA8e.

[21] "Formal Verification and Ethereum," https://ethereum.stackexchange.com/questions/11092/what-is-formalverification-and-why-is-it-important-for-smart-contracts.

[22] "Formal Verification for Solidity Contracts," https://forum.ethereum. org/discussion/3779/formal-verification-for-solidity-contracts.

[23] "HackerGold Bug," https://git.io/vFAl2.

[24] "Hyperledger Fabric," https://hyperledger.org/projects/fabric.

[25] "Hyperledger Fabric Mockstub," https://git.io/vFA8Y.

[26] “Inian Parameshwaran,” Personal Communication.

[27] "LLVM-based compiler for Go ," https://git.io/FPuO.

[28] "Logical bug," https://live.ether.camp/account/ ef71862273817c9e082ca2c92486c8dcdcd9356f/contract.

[29] "Loi Luu," Personal Communication.

[30] "Model arbitrary integer size," https://git.io/vFAlu.

[31] "Multiply your ether," https://etherscan.io/address/ 0xc357a046c5c13bb4e6d918a208b8b4a0ab2f2efd\#code.

[32] "Oyente: An Analysis Tool for Smart Contracts," https://git.io/vFAlX.

[33] "Oyente Results," https://raw.githubusercontent.com/oyente/ benchmarks/master/benchmark/results.json.

[34] "Parity MultiSig bug,"

http://hackingdistributed.com/2017/07/22/deep-dive-parity-bug.

[35] "Remove tx.origin \#683," https://git.io/vFA8n.

[36] "Scoping and Declarations," https://solidity.rtfd.io/en/develop/control-structures.html.

[37] "SeaHorn," https://seahorn.github.io/.
[38] "Send w/Throw Is Dangerous," http://vessenes.com/ethereumgriefing-wallets-send-w-throw-considered-harmful/.

[39] "Simple Dice," https://etherscan.io/address/ 0x237f29bbFd52C768A02980eA8D4D983a1D234eDC.

[40] “Smack," https://git.io/vFAlB.

[41] "Solidity Programming Language." https://git.io/vFA47.

[42] "StandardToken," https://git.io/vFAlg.

[43] “The DAO," https://en.wikipedia.org/wiki/The_DAO_(organization).

[44] "The DAO is kind of a mess," https://www.wired.com/2016/06/ biggest-crowdfunding-project-ever-dao-mess/.

[45] "Tx.Origin And Ethereum Oh My!" http://vessenes.com/tx-origin-and-ethereum-oh-my/.

[46] "Unchecked-Send Bug," http://hackingdistributed.com/2016/06/16/ scanning-live-ethereum-contracts-for-bugs/.

[47] “Underhanded Solidity coding," https://redd.it/4e5y30.

[48] "Units and Globally Available Variables," https://solidity.rtfd.io/en/develop/units-and-global-variables.html.

[49] "Wallet," https://etherscan.io/address/ 0xab7c74abc0c4d48d1bdad5dcb26153fc8780f83e.

[50] "Why3," http://why3.lri.fr/.

[51] "XACML," https://tools.ietf.org/html/rfc7061.

[52] J. Barnat et al., "DiVinE 3.0 - An Explicit-State Model Checker for Multithreaded C \& C++ Programs," in CAV' 13.

[53] L. Bauer et al., "Composing Security Policies with Polymer," in PLDI 05 .

[54] K. Bhargavan et al., "Formal Verification of Smart Contracts: Short Paper," in PLAS '16.

[55] N. Bjørner et al., "Program Verification as Satisfiability Modulo Theories," in SMT '12.

[56] A. R. Bradely, "SAT-based Model Checking without unrolling," in VMCAI 2011.

[57] M. Castro et al., "Practical Byzantine Fault Tolerance," in OSDI '99.

[58] K. Delmolino et al., "Step by Step Towards Creating a Safe Smart Contract: Lessons and Insights from a Cryptocurrency Lab." in FC ' 16.

[59] U. Erlingsson et al., "IRM Enforcement of Java Stack Inspection," in $S \& P{ }^{\prime} 00$.

[60] D. Evans et al., "Flexible Policy-Directed Code Safety," in $S \& P$ '99.

[61] A. Gurfinkel et al., "The SeaHorn Verification Framework," in $C A V$ ' 15.

[62] A. Juels et al., "The Ring of Gyges: Investigating the Future of Criminal Smart Contracts," in CCS '16.

[63] A. Komuravelli et al., "SMT-based Model Checking for Recursive Programs," in $C A V ' 14$.

[64] A. E. Kosba et al., "Hawk: The Blockchain Model of Cryptography and Privacy-Preserving Smart Contracts," in $S \& P$ ' 16.

[65] L. Lamport, "The Part-time Parliament," ACM Trans. Comput. Syst.

[66] C. Lattner et al., "LLVM: A Compilation Framework for Lifelong Program Analysis \& Transformation," in $C G O$ '04.

[67] L. Luu et al., "Demystifying Incentives in the Consensus Computer," in $C C S^{\prime} 15$.

[68] — "Making Smart Contracts Smarter," in CCS '16.

[69] K. L. McMillan, "Interpolants and Symbolic Model Checking," in VMCAI 2007.

[70] S. Nakamoto, "Bitcoin: A Peer-to-Peer Electronic Cash System." [Online]. Available: https://bitcoin.org/bitcoin.pdf

[71] D. Ongaro et al., "In Search of an Understandable Consensus Algorithm," in USENIX ATC'14.

[72] Z. Rakamarić et al., "SMACK: Decoupling Source Language Details from Verifier Implementations," in $C A V$ ' 14.

[73] F. Zhang et al., "Town Crier: An Authenticated Data Feed for Smart Contracts," in CCS '16.

[74] J. Zhao et al., "Formalizing the LLVM Intermediate Representation for Verified Program Transformations," in POPL '12. 